\title{
Super-exponential extinction of radiation in a negatively-correlated random medium
}

\author{
Raymond A. Shaw ${ }^{1}$ Alexander B. Kostinski \\ Daniel D. Lanterman ${ }^{2}$ \\ Department of Physics, Michigan Technological University, Houghton, MI 49931, \\ $U S A$
}

\begin{abstract}
It was shown in recent work that spatial correlations among obstacles of a random, absorbing medium can lead to slower-than-exponential (sub-exponential) extinction of radiation with propagation distance. Exponential decay, described by the BeerLambert law, arises in a special case when the medium contains no correlations. A third possibility, examined here, is that of negative correlations which can lead to faster-than-exponential (super-exponential) extinction. Using a Monte Carlo approach, we confirm that sub-exponential decay occurs when the volume-averaged pair correlation function is greater than zero at the scale of interest and that the Beer-Lambert law is recovered when correlations vanish. We also find that when the volume-averaged pair correlation function is negative, super-exponential extinction with propagation distance occurs. These results are of special interest to the problem of radiative transfer in cloudy atmospheres, where the pair correlation function previously has been shown to be negative and positive at different scales.
\end{abstract}

Key words: Beer-Lambert law, Spatial correlations, Poisson process

\section{Introduction}

Exponential decay is ubiquitous in nature. A prominent example is the BeerLambert law describing the exponential attenuation of radiation in a homogeneous random medium. Indeed, this law is of central importance in fields such as atmospheric radiative transfer. There are certain physical scenarios, however, where deviations from the Beer-Lambert law are possible — Specifically,

1 Corresponding author: rashaw@mtu.edu

2 Now at Department of Physics, University of Maryland 
Kostinski [1] showed theoretically that correlations in the positions of particles in a random medium can lead to slower-than-exponential (sub-exponential) extinction of intensity with propagation depth. While related results were obtained earlier by others (e.g., $[2,3,4,5,6,7]$ ), the framework introduced in [1], namely that of the Poisson process and pair correlation function from the fundamental theory of stochastic processes, is especially convenient for further exploration of this phenomenon.

In particular, the possibility of sub-exponential extinction of radiation in a positively-correlated medium leads us to ask, how do negative correlations affect light extinction? The approach introduced in [1] allows us to examine this question, if not analytically as in the original work, then computationally. Indeed, the pair correlation method is ideally suited for use in a Monte Carlo model and that is the approach taken here. For example, we consider the attenuation of radiation in a medium consisting of perfectly absorbing particles. Holding the mean number density $n$ and cross section $\sigma$ of particles constant, we explore the effect of correlations (both positive and negative) in particle position on the intensity attenuation rate.

Practical applications might include radiative transfer in atmospheric clouds, where particles positions are observed to be correlated both negatively and positively [8]. For example, a turbulent mixing zone characterized by a $-5 / 3$ scalar spectrum may possess strong positive correlations in particle position. On the other hand, sedimenting particles in a laminar fluid possess strong negative correlations in particle position [9], and an atmospheric example might be droplets in a calm fog. It also is a simple matter to conceive of macroscopic cloud geometries that would possess either negative or positive correlations (e.g., cellular convection versus $-5 / 3$ turbulence).

Given this motivation, the aim of the present paper is to: 1) extend the original argument in [1] and argue that unlike earlier work (e.g., see [1, 2, 3, 4, $5,6,7]$ negative spatial correlations reverse the effect and yield faster-thanexponential extinction; 2) present Monte Carlo simulations which confirm theoretical conclusions for both positive and negative spatial correlations.

\section{Extinction in a negatively-correlated medium: Theory}

To begin with, we consider a random medium consisting of absorbing particles. To obtain deviations from the Beer-Lambert law, it is necessary to introduce correlations among positions of particles in the medium. However, unlike the cases previously considered, these correlations are negative so that particles are less likely to be near each other than in the case of the Poisson process. Note that, in spite of correlations, the distribution of particles is still regarded 
as statistically homogeneous so that all moments (e.g., mean and variance) are invariant with respect to the shift of origin. Examples of spatially correlated particle distributions are shown in Fig. 1, which will be discussed in more detail in Sec. 3. Briefly, the left panel shows negatively-correlated particle positions, the middle panel shows uncorrelated particle positions for reference, and the right panel shows positively-correlated particle positions.

For the radiation extinction problem we must consider two "countable" random variables: the number of particles or obstacles $k$, and the number of photon absorption events $n$. To characterize the degree of spatial correlation between particles we consider two volume elements $d V_{1}$ and $d V_{2}$ that are sufficiently small so that they can contain either 0 or 1 obstacles only and the probability of containing 2 or more particles is negligible. Because the volume elements are small, the average number of particles $\bar{k} d V$ is also the probability that a particle is in the volume element $d V$. Then, for a statistically homogeneous random field, the joint probability $P(1,2)$ of finding a particle in each of the two volumes $d V_{1}$ and $d V_{2}$ is

$$
P(1,2)=\bar{k}^{2} d V_{1} d V_{2}[1+\eta(r)]
$$

where $\bar{k} d V$ is the probability of finding a particle in $\mathrm{dV}, \eta(r)$ is the pair correlation function and $r$ is the separation distance between two volumes (statistical isotropy is implied by $\eta=\eta(r)$ ).

We see from Eq. (1) that the assumption of statistical independence of counts in non-overlapping volumes implies $\eta(r)=0$ because only in this case is the joint probability simply a product of the individual ones. However, in the presence of correlations, the conditional probability of finding the 2nd particle, given that the first one is there, is enhanced or inhibited by a factor of $(1+\eta)$. In this work, our focus is on the second possibility.

As was discussed in previous work [1], there is a relationship between the variance of particle counts in a fixed sampling volume and the spatial correlation of particles. It is given by:

$$
\frac{\overline{(\delta K)^{2}}}{\bar{K}}-1=\frac{\bar{K}}{V} \int_{V} \eta d V
$$

where as in Eq. (1), $\eta$ is the pair correlation function between particle counts in volumes $V_{1}$ and $V_{2}$ within $V, \delta K \equiv K-\bar{K}$ is the deviation from the mean count in a given volume $V$ and $\bar{K}=\bar{k} V$ where $\bar{k}$ is the local mean concentration. 
After multiplying both sides of Eq. (2) by $\bar{K}$ and rearranging we obtain

$$
\overline{(\delta K)^{2}}=\bar{K}+\bar{\eta} \bar{K}^{2},
$$

where $\bar{\eta}=V^{-1} \int_{V} \eta d V$ is the volume-averaged pair correlation function. In the Poissonian case, $\eta=\bar{\eta}=0$, and this gives the well-known result that $\overline{(\delta K)^{2}}=\bar{K}$ for a Poisson distribution. In this paper we ask: what are the effects of negative correlations on the extinction? For example, $\eta<0$ might result from a form of particle "repulsion". Then it is possible to have $\bar{\eta}<0$, which is accompanied by sub-Poissonian variance in Eq. (3). Indeed, a constraint on $\bar{\eta}$ can be derived from the fact that variance must be non-negative: $\left(\overline{(\delta K)^{2}} \geq 0\right)$ yields

$$
\bar{\eta} \geq-\frac{1}{\bar{K}} .
$$

The implication is that $\bar{\eta}<0$ is allowable at all scales and, therefore, the variance in extinction events can be smaller than expected for an uncorrelated medium.

In [1] it was argued that variance of particle counts is related to the rate of extinction of radiation. As a specific example, super-Poissonian variance arising when $\bar{\eta}>0$ was shown to result in slower-than-exponential extinction. Here, we argue that sub-Poissonian variance of obstacle number yields faster-thanexponential attenuation. To that end, we recall the relevance of the Poisson process to the Beer-Lambert law of exponential extinction. We assume perfect randomness in the distribution of obstacles so that the number of absorbed photons obeys the Poisson distribution:

$$
p_{n}(x)=\frac{\overline{n(x)}^{n} \exp (-\overline{n(x)})}{n !},
$$

where $n$ is the random number of absorbed photons in the test volume per unit time, $p_{n}(x)$ is a probability of having $n$ photons absorbed in a given volume of a layer of depth $x$, and $\overline{n(x)}$ is the mean count over many realizations as a function of the depth $x$ into the slab.

Next, we consider the photon probability of transmission (no extinction) through the layer of depth $x$. That is, we need to find $p_{0}(x)$ from Eq. (5) by setting $n=0$ ( $\bar{n}$ held constant). We obtain:

$$
p_{0}(x)=e^{-\overline{n(x)}}=e^{-x / \lambda},
$$

where $\lambda^{-1}=\sigma \bar{k}$ with $\lambda, \sigma$ and $\bar{k}$ being the mean free path, extinction crosssection per obstacle, and obstacle concentration, respectively. Now, using the 
law of large numbers to interpret $p_{0}(x)$, we can rewrite Eq. (6) as

$$
\frac{N_{t r}}{N_{\text {inc. }}}=e^{-x / \lambda}
$$

which is the stochastic equivalent of the Beer-Lambert law. Here, $N_{\text {inc }}$ and $N_{t r}$ stand for the (large) number of incident and transmitted photons, respectively and $x / \lambda$ is the unitless optical depth.

In Eq. (5) we used the fact that the number of absorbed photons (at a given location) is Poisson distributed because the number of obstacles is Poisson distributed. If, however, the number of obstacles is not Poisson-distributed, then $p_{n}(x)$ will also change. For example, if the variance in the number of obstacles decreases, so does the variance in the number of absorbed photons $n$. This means that the distribution $p(n)$ gets narrower (only 'well-behaved' densities are considered for the moment). Qualitatively, we expect that $p(0)$ will decrease as well because a narrower pdf will have 'lower tails'. Therefore, at a fixed mean free path, the numerical value of the narrow (sub-Poissonian) pdf produces lower $p(0)$ than does the Poissonian case. Furthermore, the argument holds at any $\bar{n}$ which, in turn, depends linearly on $x$, penetration distance into the medium. Hence, we conclude that negative correlations yield fasterthan-exponential extinction. That this is indeed so is confirmed by evidence obtained from direct and simple Monte Carlo simulations described in the next section.

\section{Monte Carlo model and results}

To test the theory outlined in the previous section, we built a straightforward Monte Carlo model that performs two functions. First, the model generates a random distribution of particle positions, which may be spatially correlated: this gives us variable $k$. Second, the model calculates a distribution of photon absorption events by "shooting" photons through the distribution of particles: this gives us variable $n$. We will consider these two functions in turn.

Three different types of particle distributions are shown in Fig. 1. Each panel is a thin slice (slice thickness is $1 / 10$ th the width shown) taken from one realization of particle positions generated by the model. The middle panel shows particle positions that are uncorrelated at all scales, or Poisson distributed. The Poisson distribution is made by selecting each particle's coordinate independently and at random from a uniform pdf. Thus each particle's position is uncorrelated with that of any other. The left panel shows particle positions that are negatively correlated over a wide range of scales. Negatively correlated distributions can be made by randomly selecting particle positions inside 

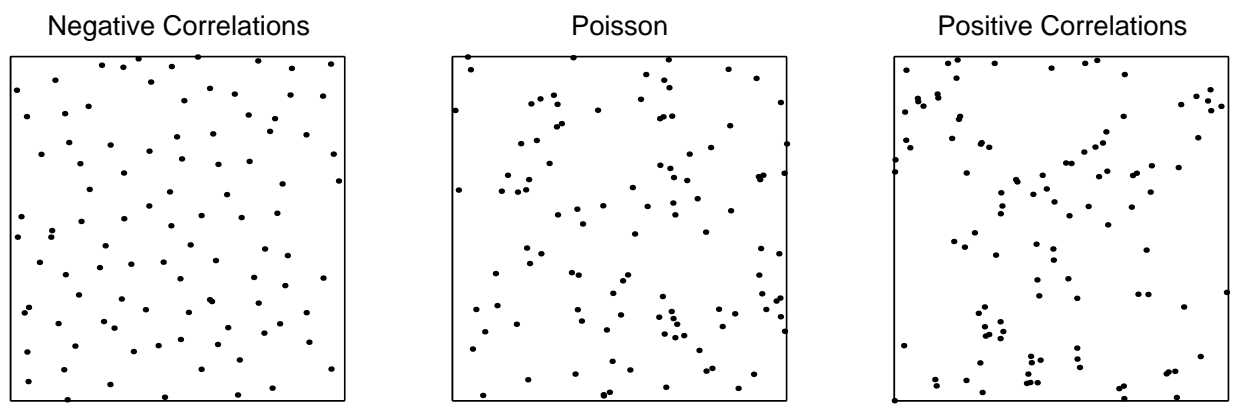

Fig. 1. Thin slices (slice thickness is $1 / 10$ th the width shown), with $n$ and $\sigma$ the same in all cases, showing particle positions in typical realizations of the Monte Carlo model. Note that in all three panels the box width is equal to one free path, defined as $1 / n \sigma$. The first panel corresponds to negatively correlated particle positions, achieved by specifying an "excluded volume" of approximately $0.8 \lambda$. The second panel is for a completely uncorrelated medium, i.e., Poisson process. The third panel is for positively correlated particle positions, achieved via an exponential conditional probability of particle position.

the box, but rejecting any position that is less than a specified distance away from any other previously placed particle. For the simulations described here, this "excluded volume" distance was $r_{\circ}=0.83 \bar{k}^{-1 / 3}$, or 0.083 times the width of the panel shown in Fig. 1. We use periodic boundary conditions to ensure that the particles do not accumulate near the edges of the box. Because this technique involves comparing each newly placed particle to all the previous ones, it is more computationally efficient to average several smaller volumes rather than one containing many particles. The right panel of Fig. 1 shows positively-correlated particle positions. Positive correlations can be achieved by using a biased pdf to select a random distance from the most recently placed particle to the next particle's location. The first particle is placed at random. In these simulations we used an exponential probability distribution with a mean step size in each direction of $0.5 \bar{k}^{-1 / 3}$, or 0.05 times the width of the panel shown in Fig. 1. Again, periodic boundary conditions were employed so that if a particle were to step outside of the box, it would be brought back in through the opposite side.

The scale-dependence of spatial correlations between particles is found by calculating the pair correlation function $\eta(r)$, where $r$ is the radial distance from one particle to another. For many realizations of the distributions in Fig. 1, we obtain the $\eta(r)$ curves shown in Fig. 2. The solid line corresponds to the Poisson-distributed particles and, as expected, $\eta(r)=0$ for all $r$. The dot-dashed line is for the positively-correlated model described previously and, indeed, confirms that $\eta(r) \geq 0$ for all $r$. The dashed line is for the "excludedvolume" model described previously, and clearly shows $\eta(r)=-1$ for $r \leq r_{\circ}$. The positive spike near $r=0.1$ is a result of particles "piling up" outside of the excluded volume, as would be expected for a near-densely-packed system. 


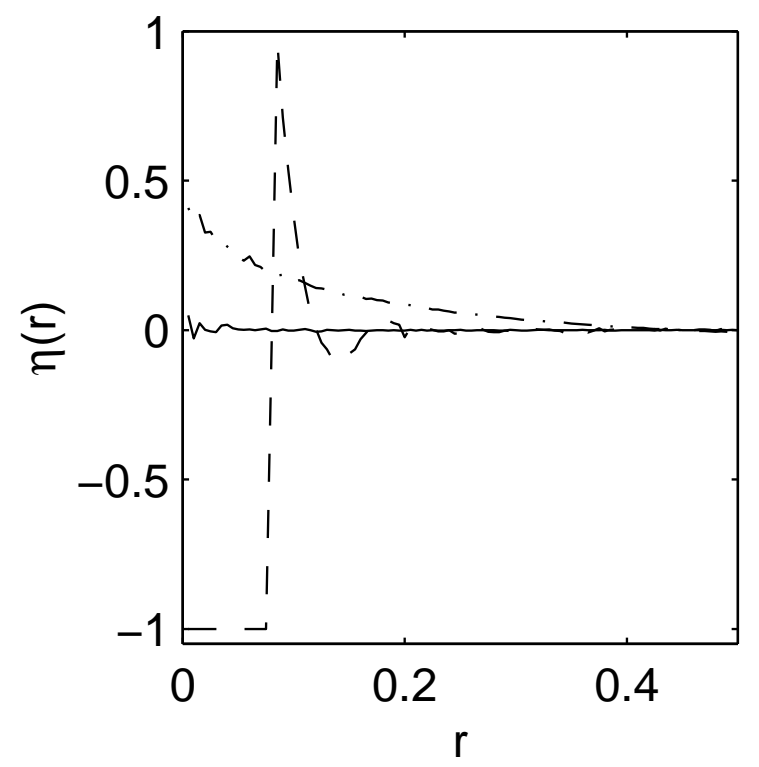

Fig. 2. The pair correlation function plotted as a function of spatial scale calculated directly from particle positions generated by the Monte Carlo model. The solid curve corresponding to no correlations shows $\eta(r)=0$ at all scales, as expected. The dot-dashed curve corresponding to the positively correlated medium shows strongly increasing $\eta(r)$ with decreasing $r$; Note that $\eta(r)>0$ for all $r$. The dashed curve corresponding to the negatively correlated medium shows $\eta(r)=-1$ for $r \leq d$, the effective particle diameter; Note that the positive fluctuations at $r>d$ do not cancel the the negative contribution when integrated to give $\overline{\eta(r)}$.

It is important to note, however, that the volume-averaged pair correlation function $\overline{\eta(r)}$ is negative at all scales so that the arguments associated with Eq. (3) still hold.

Once a particle distribution is generated, the extinction of radiation is calculated by shooting photons through the volume and keeping track of the number of photons remaining as a function of propagation depth $x$. Since the incident radiation is assumed to be incoherent, it could be represented by randomly positioned photons travelling along straight trajectories, all parallel to each other, until one passes through a particle and an absorption event occurs. All particles (obstacles) were assumed to be perfectly absorbing, so no scattering or re-emission needed to be considered. In addition, all particles were assumed to have the same absorption cross section, $\sigma$. Therefore, a photon was removed form the simulation if it passed a distance $(\sigma / \pi)^{1 / 2}$ from a particle center.

All three particle distributions had an average particle density, $\bar{k}$, of 1000 particles per unit volume. The value of $\sigma$ was then chosen to be $10^{-3}$ so that the optical depth, $\lambda=\sigma \bar{k}$ would be unity. The box was chosen to have dimensions of $1 \mathrm{x} 1 \mathrm{x} 4$. For each distribution, $10^{6}$ randomly positioned photons were sent 


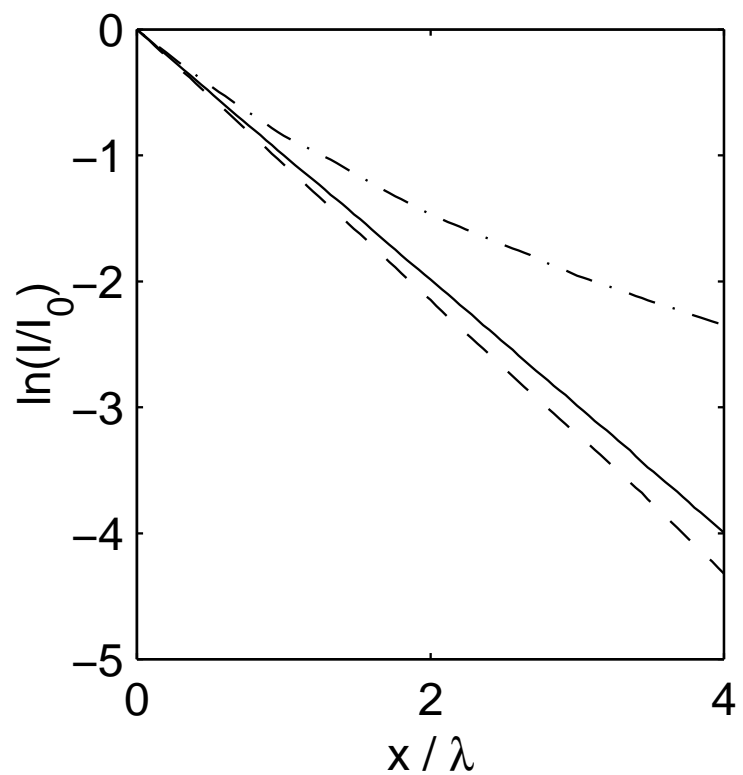

Fig. 3. Relative intensity plotted as a function of propagation depth, calculated with the Monte Carlo model described in the text. The middle curve (solid) is for the uncorrelated medium and is identical to that expected from the Beer-Lambert law. The top curve (dot-dashed), representing slower-than-exponential decay, is for the positively correlated medium. The bottom curve (dashed), representing faster-than-exponential decay, is for the negatively correlated medium.

into the particle cloud parallel to the long direction of the box. "Measurements" of the number of photons that were not absorbed were made at one hundred evenly spaced points along the box. Fifty of such simulations were then averaged to yield the extinction rate. Similar averaging could have been performed by increasing $n$ and decreasing $\sigma$ in such a manner as to keep their product constant, or by increasing the width of the box. As mentioned previously, it is computationally easier to generate smaller distributions. Finally, we note that because a particle outside the box could still potentially absorb photons that are within a distance $(\sigma / \pi)^{1 / 2}$ of one of the walls, all photons were kept this distance away from the walls. Alternatively, periodic boundary conditions could be placed on the box to enable a particle centered near one wall of the box to absorb photons near the opposite wall.

Results for the three particle distributions are shown in Fig. 3, where the logarithm of relative intensity $I / I_{\circ}$ is plotted against propagation distance normalized by the mean free path $x / \lambda$. The line types are as in Fig. 2, solid for the Poisson-distributed particles, dot-dashed for the positively correlated particles, and dashed for the negatively-correlated particles. Lack of spatial correlations faithfully reproduces the exponential decay predicted by the BeerLambert law, with $\lambda^{-1}=\bar{k} \sigma$. Extinction of radiation propagating through the positively-correlated particle distribution is significantly slower than the Beer- 
Lambert law would predict. Given that the pair correlation function shown in Fig. 2 for this particle distribution suggests a maximum $40 \%$ deviation from the uncorrelated case, it is quite striking to see this magnitude of deviation from the Beer-Lambert law. Finally, the model confirms that for negativelycorrelated particle positions (more precisely, for $\bar{\eta}<0$ ) the rate of extinction of radiation is faster than expected from the Beer-Lambert law.

While the extinction rate shown in Fig. 3 for the negatively-correlated particle distribution is faster than the expected rate based on the particle number density and cross section, further examination reveals that after a transient regime the extinction rate does, in fact, approach negative exponential (e.g., it is a straight line in Fig. 3). This transition to exponential decay with a modified optical depth $\lambda^{*}<\lambda$, occurs at approximately the same spatial scale where $\overline{\eta(x)}$ approaches zero. In fact, a similar approach to exponential extinction, but with a modified optical depth $\lambda^{*}>\lambda$, occurs for positively-correlated particle positions characterized by a "short-range" pair correlation function. Here, short-range refers to a sufficiently rapid decay of $\eta$ so that $\overline{\eta(x)} \rightarrow 0$ at some observable $x$, unlike the example shown in Fig. 3. Qualitatively, the final exponential extinction with an "effective" cross-section results when all scales with non-zero correlation are averaged over for long photon paths. Quantitative description of this will be the subject of future work, but we conjecture here that the "effective" optical depth $\lambda^{*}$ has a functional dependence on the integral of the pair correlation function, $H \equiv \int_{0}^{x} \eta\left(x^{\prime}\right) d x^{\prime}$. We note that $H \rightarrow C$, where $C$ is a constant, when $\overline{\eta(x)} \rightarrow 0$, so that we might expect a dependence such as $\lambda^{*} \propto \lambda(1+\alpha H)$, where $\alpha$ may have some functional dependence on $\sigma, \bar{k}$, etc.

\section{Concluding Remarks}

The work presented here builds on recent work [1] showing that deviations from perfect randomness in spatial positions of obstacles constituting a random medium yield departures from the Beer-Lambert law of exponential extinction with propagation distance. For example, in [1] it was argued that super-Poissonian variance yields slower-than-exponential extinction, but neither experimental nor computational confirmation was offered. In addition, the possibility of negative spatial correlations among particles was not explicitly considered. In this paper we extended the original argument to show that negative spatial correlations reverse the trend and result in faster-than-exponential extinction of radiation. Monte Carlo simulations confirm the theoretical calculations in both regimes, showing strong sub-exponential extinction for a positively correlated medium and super-exponential extinction for a negatively correlated medium. As expected, the Monte Carlo simulations reproduce the Beer-Lambert law when correlations are absent. Finally, we have speculated 
on the emergence of a modified exponential attenuation regime valid in cases of "short-range" pair correlations. Future work is focused on demonstrating non-exponential extinction in a laboratory experiment.

\section{Acknowledgements}

We thank M. Larsen for helpful discussions. This material is based on work supported by the National Science Foundation under Grant Nos. ATM-0106271 (ABK) and ATM-9984294 (RAS).

\section{References}

[1] Kostinski AB. Extinction of radiation by a homogeneous but spatially correlated random medium. J. Opt. Soc. Am. 2001; 18:1929-1933.

[2] Barker HW. Solar radiative transfer through clouds possessing isotropic variable extinction coefficient. Q. J. Roy. Meteor. Soc. 1992; 118:11451162.

[3] Cahalan RF, Ridgway W, Wiscombe WJ, Bell TL, Snider JB. The albedo of fractal stratocumulus clouds. J. Atmos. Sci. 1994; 51:24342455.

[4] Davis A, Marshak A. Lévy kinetics in slab geometry: Scaling of transmission probability. Fractal Frontiers, M. Novak and T. Dewey, eds. (World Scientific, Singapore, 1997), 63-72.

[5] Marshak, A, Davis A, Wisombe W, Cahalan R. Radiative effects of submean free path liquid water variability observed in stratiform clouds. J. Geophys. Res. 1998; 103:19557.

[6] Marshak A, Davis A, Wiscombe W, Ridgway W, Cahalan R. Biases in shortwave column absorption in the presence of fractal clouds. J. Climate 1998; 11:431-446.

[7] Weinman JA, Harshvardhan. Solar reflection from a regular array of horizontally finite clouds. Appl. Opt. 1982; 21:2940-2944.

[8] Kostinski AB, Shaw RA. Scale-dependent droplet clustering in turbulent clouds. J. Fluid Mech. 2001; 434:389-398.

[9] Lei X, Ackerson BJ, Tong P. Settling statistics of hard sphere particles. Phys. Rev. Lett. 2001; 86:3300-3303. 